\title{
Strangulated femoral hernia: the persisting clinical trap
}

\author{
Steven D. Heys and Julie Brittenden
}

Department of Surgery, University Medical Buildings, Aberdeen Royal Infirmary, Foresterhill, Aberdeen, UK.

\begin{abstract}
Summary: Five cases of strangulated femoral hernia, all with a delay in establishing the diagnosis, are reported here. These cases illustrate the continuing difficulties experienced, both in primary care and hospital practice, in making this diagnosis despite the clinical awareness of this condition.
\end{abstract}

\section{Introduction}

The diagnosis of a strangulated femoral hernia still presents a challenge to all clinicians, general practitioners, surgeons and physicians alike. We report five cases of strangulated femoral hernia, with a significant delay in establishing the correct diagnosis, resulting in serious complications which are not associated with elective repair of a femoral hernia. ${ }^{1}$

\section{Case reports}

Failure of recognition by hospital practitioners

\section{Case 1}

A woman, aged 80 years, was admitted to hospital with a 5-day history of colicky abdominal pain, vomiting and abdominal distension. Examination confirmed the generalized abdominal distension but also revealed a tender fluctuant swelling with overlying erythema of the skin, in the groin. A diagnosis of a 'groin abscess' was made and the latter aspirated percutaneously. On aspiration small bowel contents were obtained. A surgical opinion was sought immediately and a diagnosis of a strangulated femoral hernia made.

Surgery was undertaken and via a preperitoneal approach the femoral hernia was reduced, a segment of necrotic small intestine resected and primary anastomosis performed. The skin around the aspiration site was necrotic and required excision at the original operation. A secondary suture of this area was carried out 5 days later.

Correspondence: S.D. Heys, M.D., F.R.C.S.

Accepted: 9 July 1990
After making a satisfactory initial recovery the patient died from a myocardial infarction 4 days after the second procedure.

\section{Case 2}

A 71 year old female was admitted to hospital with a history of vomiting and central abdominal colicky pain. Initial examination of her abdomen had revealed no significant abnormality but abdominal $\mathrm{X}$-rays revealed small intestinal fluid levels and a diagnosis of gastroenteritis was made. (Examination of the hernial orifices was apparently unremarkable.) The patient was managed conservatively with intravenous fluids but over the following 4 days her abdomen became progressively more distended, a tender mass was now felt in the right groin and further abdominal $\mathrm{X}$-rays revealed multiple distended loops of small intestine. A strangulated femoral hernia was diagnosed and surgical advice sought. Surgery was undertaken, again through a preperitoneal approach, and a femoral hernia of the Richter's type was identified. This was reduced but a linear band of small bowel was necrotic and was simply oversewn with interrupted sero-muscular nurolon sutures. The patient made an uneventful recovery and was eventually discharged home.

\section{Case 3}

An 80 year old female was admitted to hospital with a 3 day history of vomiting. Examination revealed no evidence of external hernia but she was dehydrated and treatment with intravenous fluids for $\mathbf{4 8}$ hours was instituted although the diagnosis was unclear at this time. At the end of this period abdominal X-rays revealed distended small intestine associated with fluid levels. The patient was 
transferred to the surgical unit where a diagnosis of small bowel obstruction was made. Clinical examination revealed no evidence of an external hernia. At operation a strangulated right femoral hernia was found (Richter's type) with necrotic small intestine. A resection and primary anastomosis were performed but post-operatively the patient's general condition deteriorated, she developed multi-organ failure and died.

\section{Failure of recognition by primary care practitioners}

\section{Case 4}

A 76 year old women presented with a 5 day history of constipation, colicky abdominal pain and vomiting. A diagnosis of constipation had initially been made but this had failed to resolve and she was therefore referred to the surgical unit. Examination revealed the abdominal distension together with a tender mass in the left groin and abdominal X-rays confirmed a small bowel obstruction. A diagnosis of a strangulated femoral hernia was made and via a preperitoneal approach the femoral hernia was reduced but a segment of necrotic small intestine was resected and a primary anastomosis performed. The patient made a good recovery without further complication.

\section{Case 5}

A 58 year old female complained of an intermittent lump in the right groin for several weeks. This patient was referred from home to the surgical unit with a 5 day history of intermittent colicky abdominal pain, vomiting and a diagnosis of gastroenteritis had been initially made. Examination revealed a tender mass in the right groin and a strangulated femoral hernia was diagnosed. At operation a Richter's type hernia involving small intestine was found. This was reduced and a linear necrotic band in the small bowel oversewn with interrupted seromuscular nurolon sutures. Following this procedure the patient made a good recovery.

\section{Discussion}

The commonest cause for intestinal obstruction in the general population as a whole is adhesions, but in patients over 75 years of age, external strangulated hernias are the commonest cause. ${ }^{2}$ Therefore, with the change in population demography, and with an increasing number of elderly patients, the number of external hernias presenting to medical practitioners will increase.

The cases reported here, all in females who were 55 years of age or older, illustrate the difficulty that surgeons, physicians and general practitioners alike may have in making the diagnosis of a strangulated femoral hernia. This has been well recognized previously by $\mathrm{Hjaltson}^{3}$ who found that in a series of 46 patients with strangulated femoral hernia, $15(32 \%)$ patients were unaware of having a hernia and in 6 of these patients $(13 \%)$ the hernia was first diagnosed at laparotomy. Thus, approximately one third of such patients do not complain of any symptoms referable to the hernia itself and a tender lump need not necessarily be present in the groin to make this diagnosis. Indeed, Case 3 in our series of patients further illustrates this point. However, most patients experience central abdominal colicky pain and vomiting and careful physical examination will reveal the findings of a small bowel obstruction.

The delay in diagnosis of these patients reported in this paper was 4.7 days. However, in 1946, in a series of patients with strangulated femoral hernia the delay in diagnosis was only 2.6 days. ${ }^{4}$ This condition is thus still proving to be a clinical pitfall and we must constantly be aware of this common clinical problem if these delays are to be minimized.

It is important to minimize the delay in diagnosis because Andrews ${ }^{5}$ has shown that if there is a delay of greater that $\mathbf{4 8}$ hours, bowel resection was required in $27 \%$ of patients and there was a mortality of $21 \%$. However, if this delay in diagnosis was less than 24 hours, a bowel resection was required in only $7 \%$ of patients and with a mortality of only $1.4 \%$.

Again, it must be re-emphasized that in all patients with abdominal pain and vomiting, a thorough examination of the hernial orifices must be carried out. However, even if a tender lump is not found in the groin, the diagnosis of strangulated femoral hernia should be considered if delay and hence potentially serious complications are to be avoided.

\section{Acknowledgements}

We would like to thank the consultants concerned for permission to report their cases and to Professor Eremin for his assistance with the manuscript. 


\section{References}

1. Devlin, H.B. Management of Abdominal Hernias. Butterworths, London, 1988, pp. 121-123.

2. Blake, R. \& Lynn, J. Emergency abdominal surgery in the aged. Br J Surg 1976, 63: 956-960.

3. Hjaltson, E. Incarcerated hernia. Acta Chir Scand 1981, 147: 263-267.

4. Jens, J. Strangulated femoral hernia: a review of 100 cases. Lancet 1943, i: 705.

5. Andrews, N.J. Presentation and outcome of strangulated external hernia in a district general hospital. Br J Surg 1981, 68: 329-332. 